\title{
Comparison of Chaos Driven PSO and Differential Evolution on the Selected PID Tuning Problem
}

\author{
Roman Senkerik ${ }^{1}$, Michal Pluhacek ${ }^{1}$, Ivan Zelinka ${ }^{2}$, Donald Davendra ${ }^{2}$, \\ and Zuzana Kominkova Oplatkova ${ }^{1}$ \\ ${ }^{1}$ Tomas Bata University in Zlin , Faculty of Applied Informatics, \\ Nam T.G. Masaryka 5555, 76001 Zlin, Czech Republic \\ ${ }^{2}$ Technical University of Ostrava, Faculty of Electrical Engineering and \\ Computer Science, 17. listopadu 15,708 33 Ostrava-Poruba, Czech Republic \\ \{senkerik, pluhacek, oplatkova, ivan.zelinka, \\ donald.davendra\} @fai.utb.cz
}

\begin{abstract}
This paper presents results of the utilization of selected discrete chaotic map, which is Dissipative standard map, as pseudo-random number generator for the differential evolution (DE) optimization algorithm and Particle Swarm Optimization (PSO) algorithm in the task of PID controller design for the selected $4^{\text {th }}$ order dynamical system. The results are compared with previously published results; both chaos driven heuristics with each other and finally the obtained results are compared with canonical PSO and DE versions, which do not utilize the chaos in the place of pseudo-random number generator.
\end{abstract}

Keywords: Differential Evolution, PSO, Deterministic chaos, PID tuning.

\section{Introduction}

These days the methods based on soft computing such as neural networks, evolutionary algorithms, fuzzy logic, and genetic programming are known as powerful tool for almost any difficult and complex optimization problem.

In the past decades, PID controllers became a fundamental part of many automatic systems. The successful design of PID controller was mostly based on deterministic methods involving complex mathematics $[1,2]$.

Recently, different soft-computing methods were used with promising results for solving the complex task of PID controller design [3]. These techniques [5-8] use random operations and typically use various kinds of pseudo-random number generators (PRNGs) that depend on the platform the algorithm is implemented. More recently it was shown that chaotic systems could be used as PRNGs for various stochastic methods with great results. Some of these chaos driven stochastic methods were tested on the task of PID controller design in [4]. In [3] it was shown that Particle Swarm optimization (PSO) algorithm is able to deal with the task of PID controller design with very good results. Following that in [9 - 12] the performance of chaos driven PSO algorithm was tested on this task with great results. 
In this paper, the influence of promising discrete dissipative chaotic system to the performance of chaos driven heuristic algorithms, which is DE and PSO, are investigated and results are compared with previously published results of both canonical and chaos driven versions of evolutionary algorithm PSO [9 - 12] and with other techniques [3,4] as well as with the canonical versions of DE (without chaotic pseudo-random number generator - CPRNG).

\section{Motivation}

Till now the chaos was observed in many of various systems (including evolutionary one) and in the last few years is also used to replace pseudo-number generators (PRGNs) in evolutionary algorithms (EAs).

This research is a continuation and extension of the previous successful initial application based experiments with chaos driven PSO and PID tuning task [9-12]. In this paper the DE/rand/1/bin strategy and PSO with inertial weight driven by Dissipative chaotic map (system) were utilized to solve the issue of evolutionary optimization of PID controller settings. Thus the idea was to utilize the hidden chaotic dynamics in pseudo random sequences given by chaotic Dissipative map system to help Differential evolution algorithm in searching for the best controller settings.

Recent research in chaos driven heuristics has been fueled with the predisposition that unlike stochastic approaches, a chaotic approach is able to bypass local optima stagnation. This one clause is of deep importance to evolutionary algorithms. A chaotic approach generally uses the chaotic map in the place of a pseudo random number generator [13]. This causes the heuristic to map unique regions, since the chaotic map iterates to new regions.

The primary aim of this work is to test, analyze and compare the implementation of different natural chaotic dynamics as the CPRNGs, thus to analyze and highlight the different influences to the system, which utilizes the selected CPRNG (including the evolutionary computational techniques).

\section{PSO Algorithm}

The PSO algorithm is inspired by the natural swarm behavior of animals (such as birds and fish). It was firstly introduced by Eberhart and Kennedy in 1995 [5]. Each particle in the population represents a possible solution of the optimization problem which is defined by the cost function (CF). In each iteration of the algorithm, a new location (combination of CF parameters) of the particle is calculated based on its previous location and velocity vector.

Within this research the PSO algorithm with global topology (GPSO) [14] was utilized. The CPRNG is used in the main GPSO formula (1), which determines a new "velocity", thus directly affects the position of each particle in the next iteration.

$$
v_{i j}^{t+1}=w \cdot v_{i j}^{t}+c_{1} \cdot \text { Rand } \cdot\left(\text { pBest }_{i j}-x_{i j}^{t}\right)+c_{2} \cdot \text { Rand } \cdot\left(\text { gBest }_{j}-x_{i j}^{t}\right)
$$

Where:

$\mathrm{v}_{i}^{t+1}$ - New velocity of the ith particle in iteration $t+1$. 
$w$ - Inertia weight value.

$\mathrm{v}_{i}{ }^{t}$ - Current velocity of the ith particle in iteration $t$.

$c_{1}, c_{2}$ - Priority factors.

pBest $t_{\mathrm{i}}$ - Local (personal) best solution found by the ith particle.

gBest - Best solution found in a population.

$\mathrm{x}_{i j}{ }^{t}$ - Current position of the $i t h$ particle (component $j$ of the dim. $D$ ) in iteration $t$.

Rand - Pseudorandom number, interval $\langle 0,1\rangle$. CPRNG is applied only here.

The maximum velocity was limited to 0.2 times the range as it is usual. The new position of each particle is then given by (2), where $x_{\mathrm{i}}^{\mathrm{t}+1}$ is the new particle position:

$$
x_{i}^{t+1}=x_{i}^{t}+v_{i}^{t+1}
$$

Finally the linear decreasing inertia weight $[14,15]$ strategy was used in this work. The inertia weight has two control parameters $w_{\text {start }}$ and $w_{\text {end }}$. The values used in this study were $w_{\text {start }}=0.9$ and $w_{\text {end }}=0.4$.

\section{Differential Evolution}

DE is a population-based optimization method that works on real-number-coded individuals [8]. For each individual $\vec{x}_{i, G}$ in the current generation G, DE generates a new trial individual $\vec{x}_{i, G}^{\prime}$ by adding the weighted difference between two randomly selected individuals $\vec{x}_{r 1, G}$ and $\vec{x}_{r 2, G}$ to a randomly selected third individual $\vec{x}_{r 3, G}$. The resulting individual $\vec{x}_{i, G}^{\prime}$ is crossed-over with the original individual $\vec{x}_{i, G}$. The fitness of the resulting individual, referred to as a perturbed vector $\vec{u}_{i, G+1}$, is then compared with the fitness of $\vec{x}_{i, G}$. If the fitness of $\vec{u}_{i, G+1}$ is greater than the fitness of $\vec{x}_{i, G}$, then $\vec{x}_{i, G}$ is replaced with $\vec{u}_{i, G+1}$; otherwise, $\vec{x}_{i, G}$ remains in the population as $\vec{x}_{i, G+1}$. DE is quite robust, fast, and effective, with global optimization ability. It does not require the objective function to be differentiable, and it works well even with noisy and time-dependent objective functions. Description of used DERand1Bin strategy is presented in (3). See [8], [16] and [17] for the description of all other strategies.

$$
u_{i, G+1}=x_{r 1, G}+F \cdot\left(x_{r 2, G}-x_{r 3, G}\right)
$$

\section{The Concept of CPRNG}

The general idea of CPRNG is to replace the default PRNG with the chaotic system. As the chaotic system is a set of equations with a static start position, we created a random start position of the system, in order to have different start position for different experiments. This random position is initialized with the default PRNG, as a oneoff randomizer. Once the start position of the chaotic system has been obtained, the system generates the next sequence using its current position. 
Generally there exist many other approaches as to how to deal with the negative numbers as well as with the scaling of the wide range of the numbers given by the chaotic systems into the typical range $0-1$ :

- Finding of the maximum value of the pre-generated long discrete sequence and dividing of all the values in the sequence with such a maxval number.

- Shifting of all values to the positive numbers (avoiding of ABS command) and scaling.

\subsection{Chaotic System for CPRNG}

This section contains the description of discrete dissipative chaotic map, which was used as the CPRNG. The direct output iterations of the chaotic map were used for the generation of the both integer numbers and real numbers scaled into the typical range for random function: $<0-1>$.

The Dissipative Standard map is a two-dimensional chaotic map. The parameters used in this work are $b=0.6$ and $k=8.8$ as suggested in [18]. The map equations are given in (4).

$$
\begin{aligned}
& X_{n+1}=X_{n}+Y_{n+1}(\bmod 2 \pi) \\
& Y_{n+1}=b Y_{n}+k \sin X_{n}(\bmod 2 \pi)
\end{aligned}
$$

The typical chaotic behavior of the utilized chaotic map, represented by the example of direct output for the variable $x$ is depicted in Fig. 1.

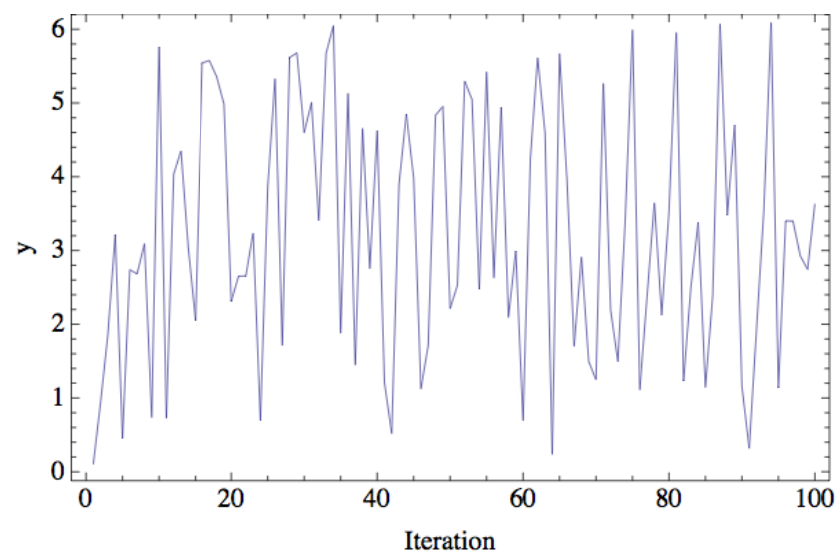

Fig. 1. Simulation of the chaotic behavior of Dissipative map (variable $y$ - line-plot)

The illustrative histograms of the distribution of real numbers transferred into the range $<0-1>$ generated by means of studied chaotic system is in Fig. 2.

Finally the Fig. 3 shows the example of dynamical sequencing during the generating of pseudo number numbers by means of studied CPRNG. 


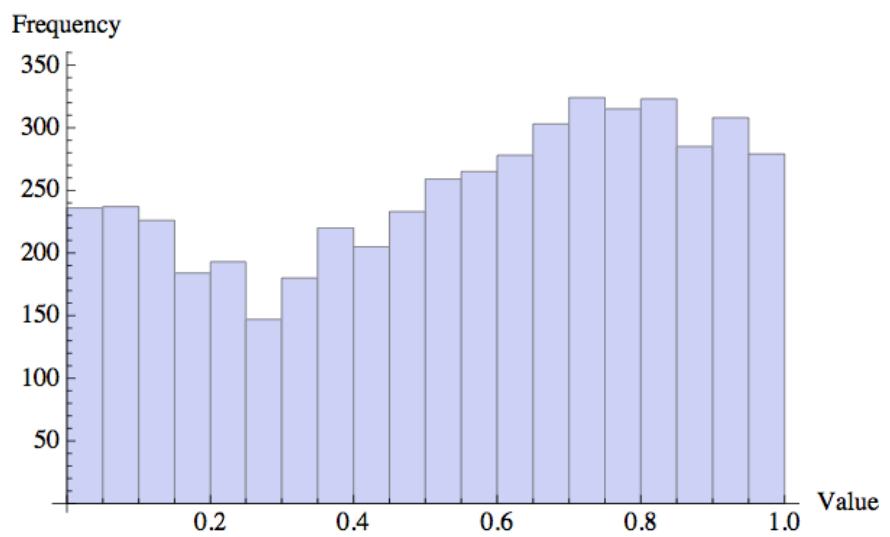

Fig. 2. Histogram of the distribution of real numbers transferred into the range $<0$ - $1>$ generated by means of the chaotic Dissipative standard map - 5000 samples

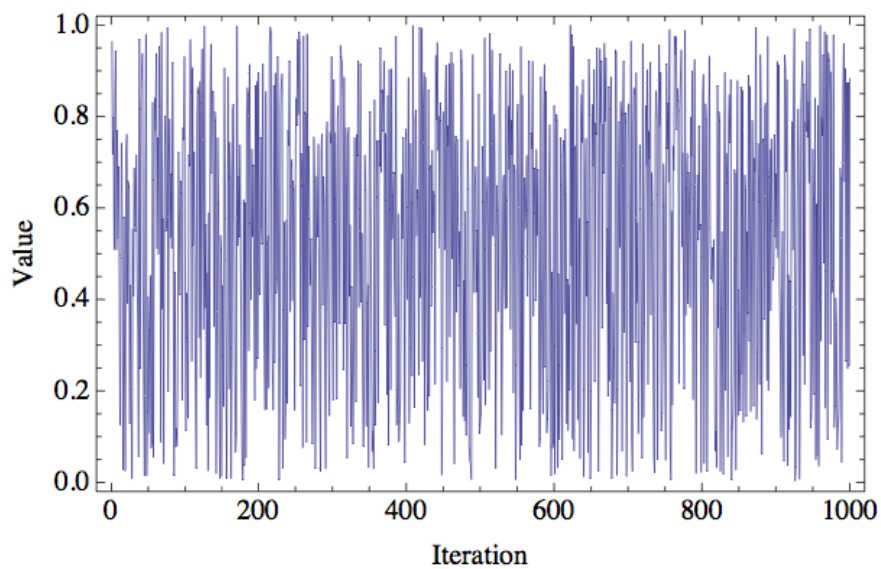

Fig. 3. Example of the chaotic dynamics: range $<0-1>$ generated by means of the chaotic Dissipative standard map

\section{Problem Design}

\subsection{PID Controller}

The PID controller contains three unique parts; proportional, integral and derivative controller [1-4]. A simplified form in Laplace domain is given in (5).

$$
G(s)=K\left(1+\frac{1}{s T_{i}}+s T_{d}\right)
$$

The PID form most suitable for analytical calculations is given in (6).

$$
G(s)=k_{p}+\frac{k_{i}}{s}+k_{d} s
$$


The parameters are related to the standard form through: $k_{p}=K, k_{i}=K / T_{i}$ and $k_{d}=$ $K T_{d}$. Acquisition of the combination of these three parameters that gives the lowest value of the test criterions was the objective of this research. Selected controlled system was the $4^{\text {th }}$ order system that is given by Eq. 7 .

$$
G(s)=\frac{1}{s^{4}+6 s^{3}+11 s^{2}+6 s}
$$

\subsection{Cost Function}

Test criterion measures properties of output transfer function and can indicate quality of regulation [1-4]. Following four different integral criterions were used for the test and comparison purposes: IAE (Integral Absolute Error), ITAE (Integral Time Absolute Error), ISE (Integral Square Error) and MSE (Mean Square Error). These test criterions (given by Eq. 8 - 11) were minimized within the cost functions for the enhanced PSO algorithm.

1. Integral of Time multiplied by Absolute Error (ITAE)

$$
I_{\text {ITAE }}=\int_{0}^{T} t|e(t)| d t
$$

2. Integral of Absolute Magnitude of the Error (IAE)

$$
I_{I A E}=\int_{0}^{T}|e(t)| d t
$$

3. Integral of the Square of the Error (ISE)

$$
I_{I S E}=\int_{0}^{T} e^{2}(t) d t
$$

4. Mean of the Square of the Error (MSE)

$$
I_{M S E}=\frac{1}{n} \sum_{i=1}^{n}(e(t))^{2}
$$

\section{$7 \quad$ Results}

In this section, the results obtained within experiments with ChaosDE and Chaos PSO algorithms are compared with each other and with previously published works $[3,4,9$ - 12]. Table 1 shows the typical used settings for the both ChaosDe and Canonical DE, whereas Table 2 contains the settings for both Chaos PSO and canonical PSO. 
Table 1. DE settings

\begin{tabular}{ll}
\hline DE Parameter & Value \\
\hline PopSize & 20 \\
F & 0.8 \\
CR & 0.8 \\
Generations & 50 \\
Max. CF Evaluations (CFE) & 1000 \\
\hline
\end{tabular}

Table 2. PSO settings

\begin{tabular}{ll}
\hline DE Parameter & Value \\
\hline PopSize & 20 \\
$\mathrm{v}_{\max }$ & $0.2 \cdot$ Range \\
$w_{\text {start }}$ & 0.9 \\
$w_{\text {end }}$ & 0.4 \\
Priority factors $c_{1}$ and $c_{2}$ & 2 \\
Iterations & 50 \\
Max. CF Evaluations $(\mathrm{CFE})$ & 1000 \\
\hline
\end{tabular}

Table 3. Comparisons of results for other heuristics $-4^{\text {th }}$ order system PID controller design

\begin{tabular}{lllllll}
\hline Criterion & $\begin{array}{l}\text { ZN } \\
\text { Step Response }\end{array}$ & Canonical DE & Chaos DE & Chaos SOMA & PSO & Chaos PSO \\
\hline IAE & 34.9413 & 12.3262 & $\mathbf{1 2 . 3 2 6 0}$ & 12.3305 & 12.3738 & 12.3479 \\
ITAE & 137.5650 & 15.1935 & $\mathbf{1 5 . 1 9 1 9}$ & 15.3846 & 16.4079 & 15.5334 \\
ISE & 17.8426 & $\mathbf{6 . 4 0 5 1 5}$ & $\mathbf{6 . 4 0 5 1 5}$ & 6.41026 & 6.40538 & 6.40516 \\
MSE & 0.089213 & $\mathbf{0 . 0 3 2 0 2 6}$ & $\mathbf{0 . 0 3 2 0 2 6}$ & 0.032027 & 0.032030 & $\mathbf{0 . 0 3 2 0 2 6}$ \\
\hline
\end{tabular}

Table 4. Statistical results of all 50 runs of Both Chaos heuristics versions

\begin{tabular}{|c|c|c|c|c|c|}
\hline DE Version & Avg CF & Median CF & Max CF & Min CF & StdDev \\
\hline \multicolumn{6}{|c|}{ ITAE Criterion } \\
\hline Chaos PSO Dissipative Map & 12.4184 & 12.3959 & 12.6140 & 12.3479 & 0.072049 \\
\hline Chaos DE Dissipative Map & 12.3274 & 12.327 & 12.3314 & 12.3262 & 0.001216 \\
\hline \multicolumn{6}{|c|}{ IAE Criterion } \\
\hline Chaos PSO Dissipative Map & 17.6267 & 17.4012 & 21.5345 & 15.5334 & 1.594303 \\
\hline Chaos DE Dissipative Map & 15.2251 & 15.2127 & 15.3212 & 15.1919 & 0.033799 \\
\hline \multicolumn{6}{|c|}{ ISE Criterion } \\
\hline Chaos PSO Dissipative Map & 6.4059 & 6.4057 & 6.4083 & 6.40516 & 0.000841 \\
\hline Chaos DE Dissipative Map & 6.40516 & 6.40516 & 6.40517 & 6.40515 & 0.000025 \\
\hline \multicolumn{6}{|c|}{ MSE Criterion } \\
\hline Chaos PSO Dissipative Map & 0.03203 & 0.03202 & 0.03206 & 0.03202 & $8 \cdot 10^{-6}$ \\
\hline Chaos DE Dissipative Map & 0.03202 & 0.03202 & 0.03202 & 0.03202 & $9 \cdot 10^{-9}$ \\
\hline
\end{tabular}


Best results obtained for each method are given in Table 3. The statistical results of the experiments for all criterions are shown in Table 4, which represent the simple statistics for cost function (CF) values, e.g. average, median, maximum values, standard deviations and minimum values representing the best individual solution for all 50 repeated runs of canonical DE and ChaosDE. The bold values within the all Tables 3 and 4 depict the best obtained results.

Furthermore an example of the step responses of the system with PID controllers designed by means of Chaos DE is depicted in Fig 4.

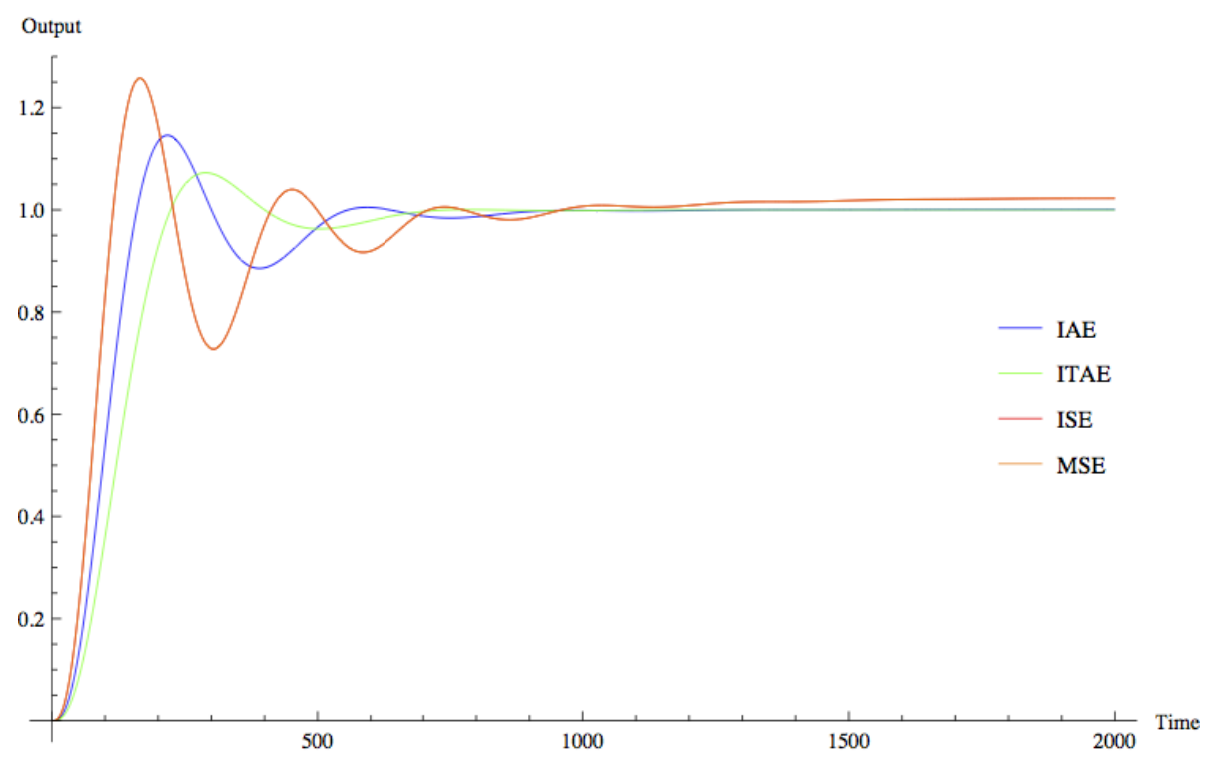

Fig. 4. Comparison of system responses $-4^{\text {th }}$ order system; Chaos DE

\section{Conclusion}

In this paper the chaotic dissipative standard map was presented and investigated over their capability of enhancing the performance of DE and PSO algorithms in the task of PID controller design.

From the comparisons, it follows that through the utilization of chaotic systems; the best overall results were obtained and entirely different statistical characteristics of CPRNGs-based heuristic can be achieved. Thus the different influence to the system, which utilizes the selected CPRNG, can be chosen through the implementation of particular inner chaotic dynamics given by the particular chaotic system. When comparing both chaos driven heuristics, DE has outperformed PSO.

Promising results were presented, discussed and compared with other methods of PID controller design. More detail experiments are needed to prove or disprove these claims and explain the effect of the chaotic systems on the optimization and controller design. 
Acknowledgements. This work was supported by Grant Agency of the Czech Republic GACR P103/13/08195S, partially supported by Grants of SGS No. SP2014/159 and SP2014/170, VŠB - Technical University of Ostrava, Czech Republic, by the Development of human resources in research and development of latest soft computing methods and their application in practice project, reg. no. CZ.1.07/2.3.00/20.0072 funded by Operational Programme Education for Competitiveness, co-financed by ESF and state budget of the Czech Republic, further was supported by European Regional Development Fund under the project CEBIA-Tech No. CZ.1.05/2.1.00/03.0089 and by Internal Grant Agency of Tomas Bata University under the project No. IGA/FAI/2014/010.

\section{References}

1. Åström, K.J.: ControlSystem Design - Lecture Notes for ME 155A (2002), http: / / www.cds.caltech.edu/ murray/courses/cds101/fa02/ caltech/astrom.html

2. Landau, I.D., Gianluca, Z.: Digital Control Systems. Communications and Control Engineering. Springer, London (2006)

3. Nagaraj, B., Subha, S., Rampriya, B.: Tuning Algorithms for PID Controller Using Soft Computing Techniques. International Journal of Computer Science and Network Security $8(4), 278-281$ (2008)

4. Davendra, D., Zelinka, I., Senkerik, R.: Chaos driven evolutionary algorithms for the task of PID control. Computers \& Mathematics with Applications 60(4), 1088-1104 (2010)

5. Kennedy, J., Eberhart, R.: Particle swarm optimization. In: IEEE International Conference on Neural Networks, pp. 1942-1948 (1995)

6. Dorigo, M., Di Caro, G.: Ant colony optimization: a new meta-heuristic. In: Proceedings of the 1999 Congress on Evolutionary Computation, CEC 1999 (1999)

7. Goldberg, D.E.: Genetic Algorithms in Search, Optimization, and Machine Learning. Addison-Wesley (1989)

8. Storn, R., Price, K.: Differential Evolution - A Simple and Efficient Heuristic for global Optimization over Continuous Spaces. Journal of Global Optimization 11(4), 341-359 (1997)

9. Pluhacek, M., Senkerik, R., Davendra, D., Zelinka, I.: Designing PID controller for DC motor system by means of enhanced PSO algorithm with discrete chaotic Lozi map. In: 26th European Conference on Modelling and Simulation, ECMS 2012, pp. 405-409 (2012)

10. Pluhacek, M., Senkerik, R., Davendra, D., Zelinka, I.: PID controller design for 4th ordersystem by means of enhanced PSO algorithm with lozi chaotic map. Paper presented at the 18th International Conference on Soft Computing, MENDEL 2012, pp. 35-39 (2012)

11. Pluhacek, M., Senkerik, R., Davendra, D., Zelinka, I.: Designing PID Controller for DC Motor by Means of Enhanced PSO Algorithm with Dissipative Chaotic Map. In: Snášel, V., Abraham, A., Corchado, E.S. (eds.) SOCO Models in Industrial \& Environmental Appl. AISC, vol. 188, pp. 475-483. Springer, Heidelberg (2013)

12. Pluhacek, M., Senkerik, R., Davendra, D., Zelinka, I.: Designing PID controller for 4th order system by means of enhanced PSO algorithm with discrete chaotic Dissipative standard map. In: EMSS 2012 24th European Modeling and Simulation Symposium, pp. 396-401 (2012) 
13. Caponetto, R., Fortuna, L., Fazzino, S., Xibilia, M.G.: Chaotic sequences to improve the performance of evolutionary algorithms. IEEE Transactions on Evolutionary Computatio 7(3), 289-304 (2003)

14. Yuhui, S., Eberhart, R.: A modified particles warm optimizer. In: IEEE World Congress on Computational Intelligence, May 4-9, pp. 69-73 (1998)

15. Nickabadi, A., Ebadzadeh, M.M., Safabakhsh, R.: A novel particle swarm optimization algorithm with adaptive inertia weight. Applied Soft Computing 11(4), 3658-3670 (2011)

16. Price, K.V.: An Introduction to Differential Evolution. In: Corne, D., Dorigo, M., Glover, F. (eds.) New Ideas in Optimization, pp. 79-108. McGraw-Hill Ltd. (1999)

17. Price, K.V., Storn, R.M., Lampinen, J.A.: Differential Evolution - A Practical Approach to Global Optimization. Natural Computing Series. Springer, Heidelberg (2005)

18. Sprott, J.C.: Chaos and Time-Series Analysis. Oxford University Press (2003) 